\title{
THINPLE - the new online Sociality is built on top of NFC-based Contacts
}

\author{
Sabrina Gaito, Christian Quadri, Gian Paolo Rossi, Matteo Zignani \\ Computer Science Dept. \\ Università degli Studi di Milano \\ Milan, Italy \\ Email: firstname.lastname@unimi.it
}

\begin{abstract}
The rapid densification of today's online social networks is progressively draining their early value of mirroring real life sociality. This paper describes THINPLE, a novel mobile computing $a p p$ aiming at building a protected and trusted online social space where the growth of the sociality of a person is only fuelled by contacts in the physical world. THINPLE exploits each physical contact, through the short radio range of Near Field Communication, NFC, to capture the explicit willingness and trustworthiness to initiate a online relationship. Moreover, through physical contacts, inanimate objects of the real world can be added to the online sociality of individuals, thus turning into practice the concept of Internet of things and people.
\end{abstract}

Keywords-Near Field Communication, Mobile social networks, Internet of things

\section{INTRODUCTION}

The social graphs underlying today's social networks are quickly densifying. This is progressively draining the value of online social networks due to the widespread presence of no worth ties. This is also raising concerns, for instance, about spam control and preservation of user's image against infringements deriving from posts issued by untrusted 'friends'. Despite the frequent encouragements to clean up personal links, the growth mechanism of today social networks is unable to ensure a trusted space where persons can reinforce their friendship and enlarge their social capital.

THINPLE is a novel mobile computing app, aiming at building a protected and trusted online social space as the mirror of the real life sociality manifested by physical encounters. By viewing the phone as the extension of ourself, THINPLE exploits each physical contact, currently through the short radio range of Near Field Communication, NFC. This is the next generation short-range wireless communication technology which enables the exchange of data between devices bought within the distance of 4 centimetres. Due to this very short communication range, it allows us to capture the explicit willingness and trustworthiness to mirror such a contact event into the personal online sociality. Besides, this feature is enforced by Android system policy which disables the NFC device when the mobile phone is in standby mode. The THINPLE's approach, unlike current social networks, perfectly recreates real life friendship creation phase as a synchronous event involving both parties. At the contact time, people agree about friendship, thus avoiding individuals getting bored about spam of friendship requests. In THINPLE, the growth of the social space of a person is only fuelled by contacts in the physical world.

This basic idea has another great and unexplored potential; through physical contacts, inanimate objects of the real world can be added into the online sociality of individuals, thus turning into practice the concept of Internet of things [1] and people. By equipping objects with NFC tags, a contact with an object explicitly adds it to our space of sociality. As the consequence, restaurants and shops, monuments and points of interest, as well as home appliances or personal objects can enter the social graph of individuals and communicate through trusted channels to provide a variety of services: from fidelity programs and advertisement to remote control and product traceability. Moreover THINPLE makes possible the creation of emerging communities around the inanimate objects. These communities are based on a shared real interest which makes itself visible through the creation of a link on the online social network.

THINPLE provides a unifying framework where people and things are seamlessly combined into a common graph. For this reason, the name THINPLE is obtained from crasis of words things and people. THINPLE is inherently a mobile application and is complemented by cloud services. It aims at the following:

- create and manage the social capital of individuals as composed of trusted people and loved objects;

- ensure a protected space where social interactions are conducted and the personal social capital can be expanded;

- enable service providers to deploy their applications on the top of THINPLE social network.

\section{THE ONLINE SOCIALITY OF THINPLE}

THINPLE adopts a novel creation and growth policy and applies it to the model of online sociality that is adopted by most of today's social networks. The THINPLE's aim is expressing the social potential of people and things by leveraging contacts in the real world to make explicit friendship requests or interest towards a thing. This contact-based growth policy allows us to create the scenario of Figure 1(a), where each person, let's say A, B, C, E and G (white nodes), is represented by a node in a graph with links to neighbor nodes associated 


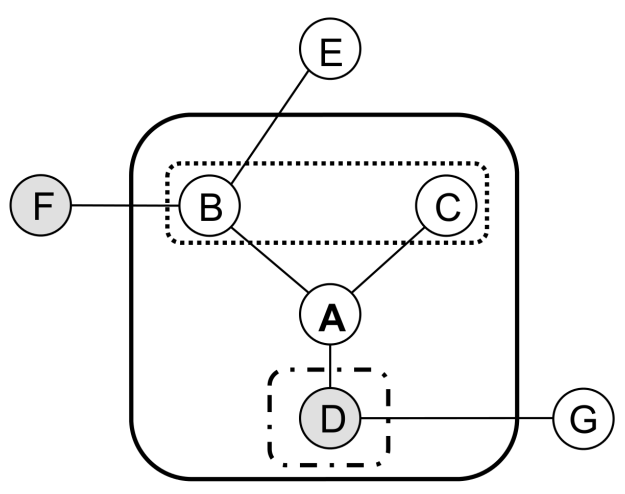

(a)

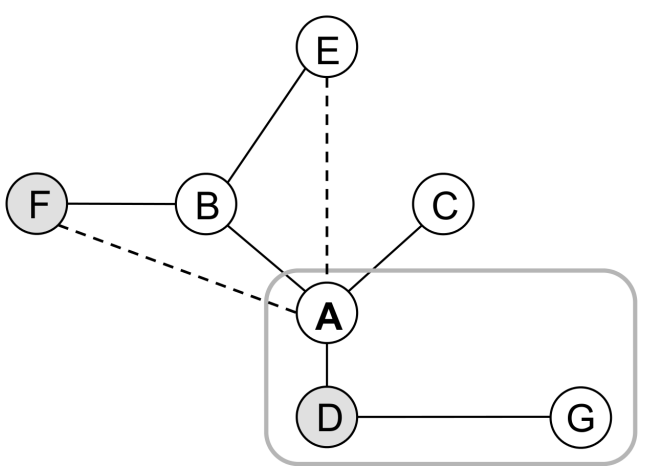

(b)

Fig. 1. An example of THINPLE graph. a) White nodes represent people and grey nodes represent things. The social capital of the node A (solid box) is the union of their human (dotted box) and environmental (dashed box) capitals. Solid lines represent links happened as a result of real encounters. b) Dashed links represent two hops potential friendships mediated by the person B, while the node (thing) D is the mediator for the community (grey box) grouping nodes $\mathrm{A}$ and $\mathrm{G}$.

to encountered friends. In this view the node neighborhood represents the real human capital of a person and mirrors his/her sociality. Inanimate objects, let's say D and F (grey nodes), surrounding a person and catching his/her interest, can also enter the graph. They become the environmental capital of a person. As a consequence each person is characterized by a social capital (solid line in Fig.1(a)) which is the union of human (dotted box in Fig.1(a)) and environmental capitals (dashed box in Fig.1(a)).

As we can see in Fig.1(b), a person can expand the borders of his/her social capital by exploiting the his/her two-hops view as follows. As mentioned before the persons' one-hop neighborhood (the social capital) is made up by things (the environmental capital) and people (the human capital). The two-hop viewing allows a user to look at the social capital of his/her human capital only; in a nutshell you can see the friends and the objects of all your friends, but you cannot see people who tapped on the same object. This choice is motivated by observing that a two-hops view of people who tapped on an object (for instance, a monument) is mostly useless (and often unaffordable). For instance, A is aware of B and C neighbors (E and F), but s/he can't see D neighbors (G). This feature allows the creation of weak connections representing potential new friendships. For instance, a node, say A, may use a weak connection (dashed link), i.e. link towards a 2hop node, to $\mathrm{E}$ to plan an encounter, that still remains the only mechanism to let E enter the A's human capital (turning the dashed into solid link). As regards environmental capital, the two-hop viewing makes easier the growing of personal environmental capital. For example, should $\mathrm{F}$ be a monument, by viewing $\mathrm{F}$ in the B's space, A may be encouraged to visit it, tap on the NFC tag of F thus letting $\mathrm{F}$ to enter his/her environmental capital.

In our design a thing's node has a functionality that a person's node has not: it can act as a mediator to form a community of people sharing the same interest about the thing (solid grey box in Fig.1(b)). Many of those could be willing to share comments. To appease this wish, a person can join the community that has been opened by the object. All communications inside a community rely on the mediation of the relevant object and are not performed through dashed links, as happening between persons.

In THINPLE the Internet of people and the Internet of things are merged and linked by relationships that model real life attitude to manage and expand personal human and environmental capital.

\section{THE FUNCTIONAL ARCHITECTURE OF THINPLE}

In this section we present the functional architecture of THINPLE platform. The architecture has been designed in order to easily allow the service providers to deploy their application on the top of THINPLE platform. To accomplish this task we build the THINPLE architecture maximizing the modularity and reusability of the different components. Another design guideline is the efficient management of the social graph, representing the social network, and the contents associated to nodes and their relationships.

At the moment, THINPLE relies on a centralized social graph. In fact, our first aim is the construction of a new sociality leaving privacy concerns and disintermediation issues to a future phase. THINPLE has two functional components: THINPLE-mobile and THINPLE-cloud (Figure 2).

\section{A. THINPLE-Mobile}

The THINPLE-mobile component includes the following three modules:

- Contact Manager - this component receives the NFC contact descriptor and generates the associated event that is described by the tuple <nodeID, timestamp, [public key], [encounter ticket] $>$. While the timestamp is generated locally, all the other (mandatory and optional - in brakets) data are exchanged at contact time. Both persons and things are identified by a unique nodeID, obtained in a similar way of IMSI number of mobile phones and is composed of three parts: type, country code, provider 


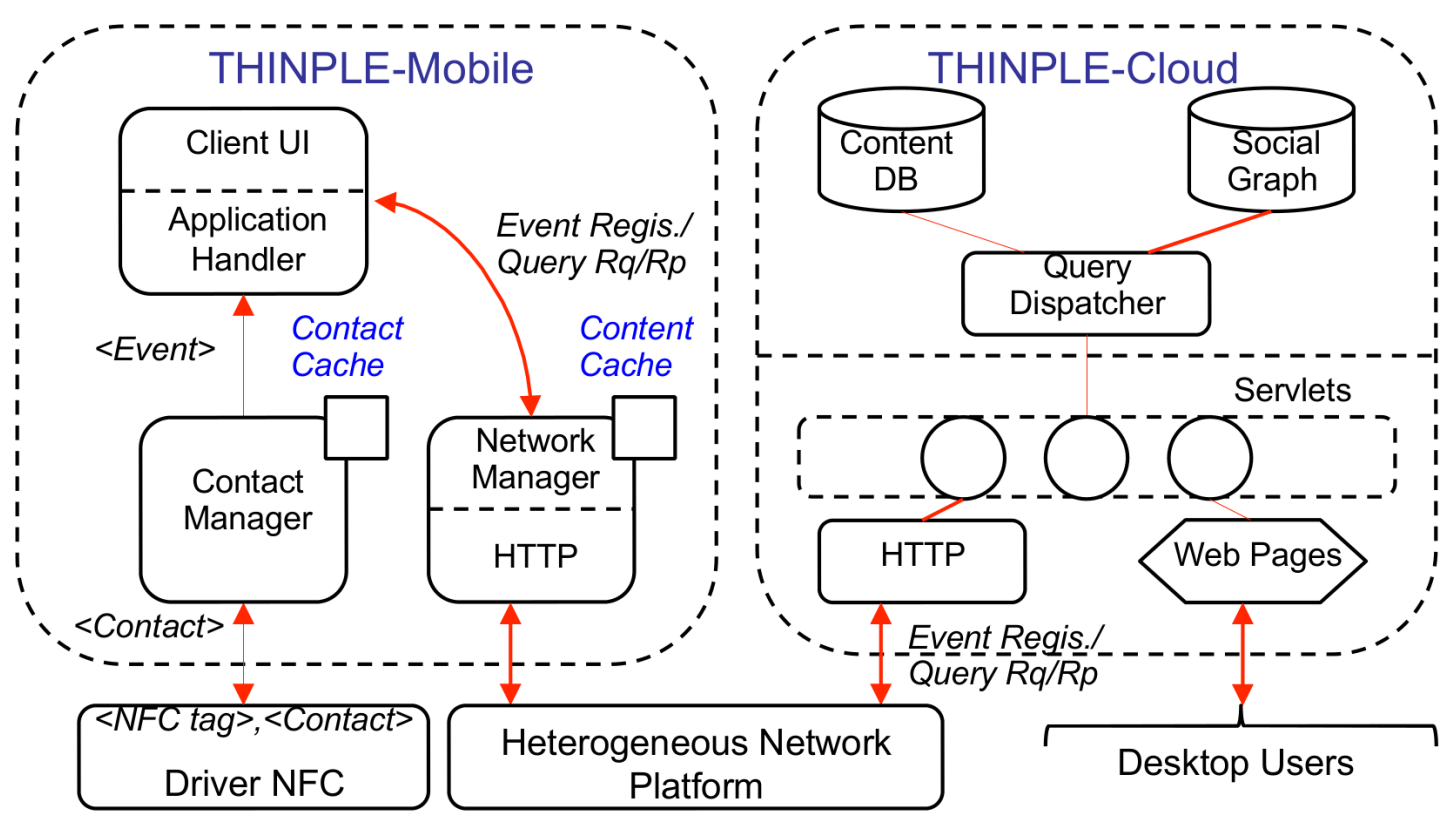

Fig. 2. The architecture of THINPLE-mobile and THINPLE-cloud.

code, tag number. The first is used to distinguish people and things; the second is unused at the moment and accounts for future possible organization by region; the third is set to 001 for THINPLE and could be useful when different service providers will be using the same THINPLE platform. The last is a progressive integer. This nodeID uniquely identifies it inside the social graph on the THINPLE-cloud.

The Contact Manager has to manage two types of contact: among people, and among people and things. In the first contact type, the mobile phone is reader and sender of contact descriptor at the same time. In order to successfully complete the exchange, both devices have to simultaneously send their contact descriptors. Unfortunately, the end-devices of a NFC channel are rarely synchronized. Due to the current Android NFC policy, in such an asynchronous exchange the first sending device happens to block the sending of the other one. To prevent this event, the Contact Manager receiving the first NFC contact descriptor starts a timer. Should the timer expire without closing the exchange, the Contact Manger transparently opens a Bluetooth channel to complete it. At this point, both peers are autonomously able to update their human capital on THINPLE-Cloud. In the second contact type, the mobile phone is acting as NFC-reader and it has the responsibility to update the users's environmental capital. Each contact event is locally cached to avoid multi event occurrences due to multiple tapping;

- Network Manager - It realizes the Internet (cellular and WiFi networks) connectivity to THINPLE-Cloud. The network channel (WiFi or $3 \mathrm{G}$ ) is automatically selected by Android operating system. The Network Man- ager negotiates with THINPLE-Cloud which contents to download. The Network Manager sends to server the current channel condition, using the system API, and the THINPLE-Cloud selects the contents to send to mobile, according to its policy. For instance, if we are in presence of a WiFi network (a very good network condition with respect to $3 \mathrm{G}$ network), the server decides to send all contents to mobile (text, images, video, and more). If we are in presence of $3 \mathrm{G}$ network, the server decides to send to mobile the lighter part of contents (text and small images), thus improving the responsiveness of the application. The content transfer is operated through HTTP/HTTPS sessions. A local cache maintains the most recent downloaded contents to avoid unnecessary network access may cause waste of energy. In absence of radio connectivity, pending requests to THINPLE-Cloud are temporarily queued locally and and they will be sent to THINPLE-Cloud as soon as the connectivity became available.

- Client Application - the component has two modules. The Application Handler provides a unifying API interface towards the module Client GUI to ensure transparency with respect to the underlying communication channels. The Application GUI implements the user interface of THINPLE. Moreover it is responsible to orchestrate the below components.

\section{B. THINPLE-Cloud}

On the server side, the THINPLE-cloud component includes:

- Server applets - A set of JAVA Servlets manages all interactions with the client side, through HTTP/HTTPS 
sessions, and with desktop users. Each Servlet may generate requests to the Query Dispatcher module whenever data have to be retrieved. The interface between the two modules has been designed to ensure layer independence.

- Social Graph - This component is responsible to manage the social network using a Graph Database instead a Relational Database in order to efficiently navigate into the social graph.

- Content DB - This module manages a Relational Database containing all contents regarding the node (name, photos, ...) and the relationships (date of meeting, shared content, meeting place, ...).

- Query Dispatcher - According to the type of the query this module routes requests to the Social Graph (for instance, when it is required to navigate the graph) or to the Content DB (for instance, to retrieve the contents associated to a given object), and forwards replies to the proper Servlet. The contents associated to graph nodes and social graph are kept separated to satisfy scalability and performance issues.

\section{ENABLING NEW MOBILE APPLICATIONS}

The aggregation of people and things into a unifying social network is the richness of THINPLE and enables or improves a variety of challenging mobile applications and services beyond the traditional services of online social network. It is in fact very simple to imagine a variety of application scenarios centered around things, as briefly shown in the sequel.

- Example 1 - Let's imagine that F is actually the bike of B. This enables $\mathrm{F}$ to be present into the B's space with general information and photos, but also to deliver workout data for comprehensive off line analysis (see, for instance, GarminConnect).

- Example 2 - Let's now assume that the object D is a pub, or an art gallery or a temporary shop. Each person who visited it at least once has a link to it and all friends have a two-hops view of it. The owner of D can advertise a forthcoming event that can be viewed two-hops apart. To open a window onto the future, THINPLE powers the "I'll go" button that enables interested people to state the willingness to attend the event. This would be useful not only to the owner of $\mathrm{D}$, but also to favor people aggregation.

In the following subsections we provide some more details about two novel mobile applications we are working on and that are currently in a prototype stage.

\section{A. Innovating the Grand Tour approach to travels}

The approach to cultural travels has been unchanged for almost three centuries now. Both modern and Grand Tour travelers mine their voyage information from books and guides. Today, several institutions rely upon the web to promote their cultural heritage; the web, however, is a great information source mainly while preparing the trip. On the

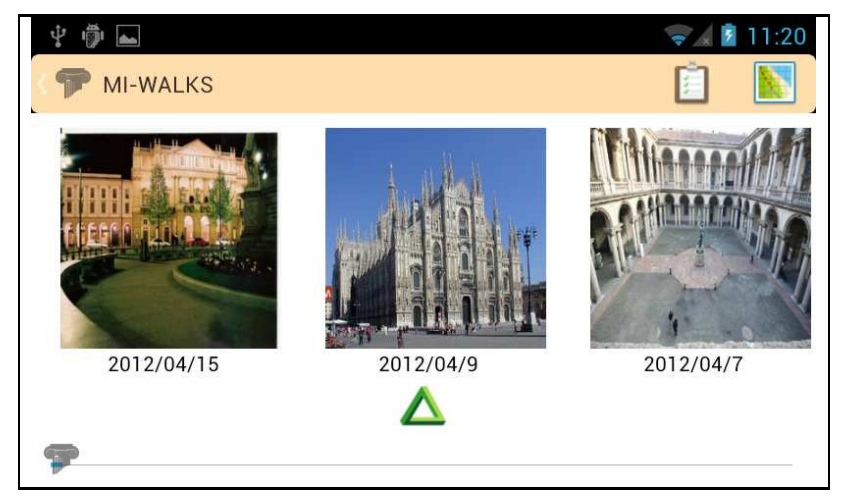

Fig. 3. Timeline of encountered monuments.

other hand, the attempts to port guides on mobile devices went almost flat as unable to add value in terms of both usability and functionalities. There is nothing that can be done to complement traditional travel supports and to improve the travel experience? We believe that innovation efforts could be certainly addressed to improve usability and to satisfy all social needs that the travel experience generates. THINPLE can do a lot in many directions. Let's assume that $\mathrm{D}$ is a monument with NFC tag, relevant contents stored into the DB and a node created in the THINPLE graph. When A taps on the D's tag, s/he gets direct access to D's contents and, at the same time, D shows up in the A's social space thus enabling A to share with friends contents associated to $\mathrm{D}$ as well as to join the D's community. The main benefits achieved by using the THINPLE approach with monuments are the following:

- Usability - in this case, tapping on the NFC tag gives direct access to information relevant to the object a person has in front. A sort of autonomic content retrieval directed by pointing the object in front of you. A natural and simple gesture.

- Human sociality - the travel is a life experience with known impact on the sociality of a person. THINPLE helps users to automatically enrich their social capital with contents about objects and friends, avoiding them using different apps to publish life experiences. THINPLE can be useful to share these experiences, to stimulate friends to do the same and to freely discuss about something you like.

- Diary - THINPLE automatically generates the user travel diary mirroring the real encounters (things and people) happening during user travel.

- Statistical analysis - All contact events with D are stored in the THINPLE-cloud and provide the institution promoting a cultural legacy with a rich dataset for operating its monuments and the communication channels to promote them.

\section{B. Product traceability and control}

The information system of the manufacturer of a domestic boiler can perfectly trace all the components that have been assembled to produce the final product, but it is generally 
unable to track the last hop to the customer which installs it at home. This makes difficult, for instance, to manage product recalls and to assist the customer for maintenance. Should the customer tap on the product tag when it is delivered at home, the product immediately enters the owner's environment and the missing link is created. At this point, we can envision a step forward; in fact, by providing the appliance with some communication capability and by means of some synoptic panel the customer will be able to remotely control his own boiler. This latter is an example in which the exchange of public keys at contact time (see Section III) could be needed to secure the communication between the two parties.

\section{IMPLEMENTATION DETAILS}

The prototype of THINPLE-mobile is available on Android platform Version 4.0 or greater using a mobile phone equipped with NFC and Bluetooth devices. The contact manager exploits the features of Android Beam to exchange data between two mobile devices, and complements with Bluetooth if required as described in Section III.

The prototype of THINPLE-cloud is developed using the following technologies: Neo4j [2] versions 1.6 for Social Graph, PostgreSQL 8.4 [3] for Content DB, and JavaServer Pages (JSP) for servlets implementation.

\section{RELATED WORK}

The scientific community has been attracted to study the human sociality in order to better understand the mechanisms leading it. The knowledge about these can be exploited to offer services leveraging the social information, and to guide the design of new network paradigms as opportunistic networks, which exploit unplanned encounters to route data among the devices [4], [5], [6]. In the last years many efforts have been done to identify the real human encounters. They have been inferred using the devices (Bluetooth, GPS, Wi-Fi) proximity [7], [8], [9], [10] or self reported surveys [11], [12]. However these experiments only capture the people proximity, but lack in capturing the explicit willingness of a meeting. Smartphones equipped with NFC device allow us to overcome this limitation. Up to now NFC has been exploited to exchange personal information or content sharing between people [13], and between people and object, as for payments. To the best of our knowledge this is the first social network based on real encounters, and including both people and object.

\section{CONCLUSIONS}

This paper proposes a new vision of the Internet of Things and People where individuals and smart objects build together a trustable social network. To turn into practice this paradigm, we present a mobile-cloud platform, THINPLE, which allows users to be part of an online social space where the growth of their sociality is only fuelled by contacts in the physical world, both with people and inanimate objects. It will provide to the research and industrial community a new, very rich but closely tied to real word, network on the top of which to deploy the multitude of services people are just beginning to envision.

\section{ACKNOWLEDGMENT}

The authors are grateful to Fabio Beccari, Gian Marco Sibilla, Silvio Bernardinello, Matteo Tanfoglio and Miguel Patrick Rosales Rojas for their skilled and efficient work in developing the THINPLE prototype. This work was funded by the Italian Ministry for Instruction, University and Research under the PRIN PEOPLENET (2009BZM837) Project.

\section{REFERENCES}

[1] The Internet of Things: A survey, vol. 54, no. 15, 2010. [Online]. Available: http://www.sciencedirect.com/science/article/pii/S1389128610001568

[2] Neo4j. [Online]. Available: http://neo4j.org/

[3] Postgresql. [Online]. Available: http://www.postgresql.org/

[4] A.-K. Pietil"ainen and C. Diot, "Dissemination in opportunistic social networks: The role of temporal communities: the role of temporal communities," in MobiHoc'12: Proceedings of the Thirteenth International Symposium on Mobile Ad Hoc Networking and Computing, June 2012.

[5] M. Pitkanen, A. Passarella, S. Giordiano, F. Legendre, T. Spyropoulos, J. Ott, T. Karkkainen, M. Conti, D. Puccinelli, M. May, N. Hegde, K. Hummel, and S. Trifunovic, "Scampi: Service platform for social aware mobile and pervasive computing," in Proc. ACM Mobile Cloud Computing Workshop, 2012.

[6] C. Quadri, D. Maggiorini, S. Gaito, and G. P. Rossi, "On the scalability of delay-tolerant routing protocols in urban environment," in IFIP Wireless Days, vol. 1, 2011, pp. $1-6$.

[7] crawdad. [Online]. Available: http://crawdad.cs.dartmouth.edu/

[8] N. Eagle, A. Pentland, and D. Lazer, "Inferring friendship network structure by using mobile phone data," Proceedings of The National Academy of Sciences, vol. 106, pp. 15 274-15 278, 2009.

[9] S. Gaito, E. Pagani, and G. P. Rossi, "Strangers help friends to communicate in opportunistic networks," Computer Networks, vol. 55, no. 2, pp. 374-385, 2011.

[10] S. Gaito, E. Pagani, G. P. Rossi, and M. Zignani, "Sensing multidimensional human behavior in opportunistic networks," in MobiOpp'12 - Proceedings of the 3rd ACM International Workshop on Mobile Opportunistic Networks, 2012, pp. 89-90.

[11] T. Hossmann, F. Legendre, G. Nomikos, and T. Spyropoulos, "Stumbl: Using facebook to collect rich datasets for opportunistic networking research," in WOWMOM, 2011, pp. 1-6.

[12] Realitymining. [Online]. Available: http://reality-media.mit.edu/

[13] B. Dodson, I. Vo, T. Purtell, A. Cannon, and M. Lam, "Musubi: disintermediated interactive social feeds for mobile devices," in Proceedings of the 21st international conference on World Wide Web, ser. WWW '12. New York, NY, USA: ACM, 2012, pp. 211-220. [Online]. Available: http://doi.acm.org/10.1145/2187836.2187866 